\title{
Editorial: Design and Control of Adaptive Civil Structures
}

\author{
Gennaro Senatore * and lan F. C. Smith \\ Applied Computing and Mechanics Laboratory (IMAC), School of Architecture, Civil and Environmental Engineering (ENAC), \\ Swiss Federal Institute of Technology (EPFL), Lausanne, Switzerland
}

Keywords: adaptive structures, adaptive facades, sustainable design, structural optimization, structural sensing, structural control, vibration control, shape morphing

\section{Editorial on the Research Topic}

\section{Design and Control of Adaptive Civil Structures}

The environmental impact of buildings and civil infrastructure has become an important topic owing to significant non-renewable material use and the greenhouse gas emissions (GHG) that are required for sourcing, extraction, component manufacturing, transport, fabrication and operation. This Research Topic investigates new design strategies, control methods and applications for structures that adapt to loading events and other environmental actions through sensing and actuation. Actuation is most often implemented at the component scale. Strategic integration of active components enables modification of structural behavior under loading to fulfill control objectives. When included during design, adaptation enables a significant improvement in performance because the structure can sense and react to change of external stimuli and thus it can operate optimally under different conditions.

Adaptation is carried out in various ways. For example, the inherently adaptive features of nature-

OPEN ACCESS

Edited and reviewed by: Branko Glisic,

Princeton University, United States

*Correspondence:

Gennaro Senatore

gennarosenatore@gmail.com

Specialty section:

This article was submitted to Structural Sensing, Control and Asset Management, a section of the journal

Frontiers in Built Environment

Received: 23 June 2021 Accepted: 29 June 2021 Published: 09 July 2021

Citation:

Senatore $G$ and Smith IFC (2021) Editorial: Design and Control of Adaptive Civil Structures. Front. Built Environ. 7:729752. doi: 10.3389/fbuil.2021.729752 inspired compound elements are integrated into structures to reduce the response under seismic excitations and to mitigate the onset of instability in long-span structures (Chenaghlou et al.). Variable stiffness and damping properties of components made of viscoelastic material (e.g., shape memory polymers) have been investigated in the form of structural joints for vibration control. Actuation through thermal energy causes a significant stiffness reduction and a parallel increase of damping which results in the shift of the structure natural frequencies and an increase in damping ratios. This semi-active control strategy is effective for multi-story buildings and bridges under various excitations such as pedestrian/vehicular traffic and earthquakes (Wang et al.). In Kelleter et al. numerical and experimental studies are carried out on concrete beams equipped with multiple disc-shaped fluidic actuators. Controlled expansion of the fluidic actuators enables the reduction of bending-induced stress and compensation of displacements. Adaptation has been investigated to design multifunctional façade components. In Neuhaus et al. experimental studies have been carried out on the integration of ionic electroactive polymer actuators (IEPA) in adaptive membrane building skins. Actuation of small apertures provides ventilation control and humidity regulation. At the same time, the embedded devices work as sensors for load monitoring. Experimental studies show the potential and limitations of IEPA for adaptive building skins.

Integration of linear actuators into truss and frame structures enables the implementation of many control strategies. Generally, controlled length changes of the actuators allow for the internal force flow and the structural shape to be manipulated to fulfill a control objective. In Cai et al. this strategy has been employed for shape control as well as locomotion of tensegrity structures. A formulation based on genetic algorithms and dynamic relaxation is developed to determine optimal control commands for shape control of a double-layered tri-prism tensegrity structure, as well as optimal gaits and motion paths of a sixstrut locomotive tensegrity structure. 
In the context of civil structures, force and shape control through linear actuators have been employed to counteract the effect of loading through stress homogenization and compensation of displacements. In Steffen et al. the use of actuation influence matrices is employed to evaluate how forces and displacements are modified by the length change of actuators that are installed either in series or in parallel with structural elements. This analysis quantifies the effect of the structural topology on actuator efficacy with regard to force and displacement compensation for optimal actuator placement. In Modano et al. a method based on influence matrices is formulated to determine optimal tension forces in the stays of through-type arch bridges with suspended deck. The objective is to obtain a target moment distribution over the deck that mitigates stress under loading. Owing to the reduced model formulation, this process has potential for a real-time control strategy using the bridge stays as active tendons. In Geiger et al. a formulation for optimal element sizing and computation of control commands is applied to design truss and frame structures equipped with linear actuators. The actuator placement is predetermined and actuators are installed in series with the structural elements. Both truss and frame structures are studied. Case studies show significant mass savings can be achieved through control of displacements for stiffness governed structures, confirming findings of previous work on similar adaptive structural types. Numerical and experimental studies are carried out in Wagner et al. on optimal load compensation of a 1:18 scaled high-rize building model equipped with actuators installed in parallel with some of the columns and in series with some of the diagonal bracing elements. The displacements are measured through a camera system. Actuator failure scenarios are simulated to quantify control performance degradation and to test the reconfiguration capability of the controller.

In Reksowardojo et al. experimental studies are carried out on a small-scale prototype structure that is designed to counteract the effect of loading through shape morphing. Instead of having one unique geometrical configuration that is the best fit to resist the envelope of peak loads, the structure is designed to "morph" into a shape that is optimal to take each load occurrence. The optimal shape changes as the load changes. Load control through shape adaptation enables significant stress homogenization so that the design is no longer dominated by peak demands. This way, material utilization is maximized and thus the embodied energy is reduced. A similar approach is taken in Sachse et al. that presents heuristic methods useful to identify a subset of external or internal actuator locations that enable control of the structure through a required motion path between two geometric configurations. An optimal deformation path between an initial undeformed geometry and a prescribed deformed configuration is identified. Actuator placement is carried out through heuristics that minimize the elastic energy (i.e., cost of deformation) integrated over the optimal motion path.

Generally, structural adaptation enables a significant reduction of material input since the structure no longer relies exclusively on passive load-bearing resistance. However, adaptation might require significant operational energy input. In Senatore and Reksowardojo an integrated structure-control optimization process is formulated to design minimum energy adaptive structures. The design objective is whole-life energy minimization including a share embodied in the material and a share for the operation of the active system. Minimum energy solutions are obtained through combined element sizing and actuator placement optimization. Actuators are installed in series with structural elements and at the supports. Four control strategies are compared through simulations on a slender high-rize structure and an arch bridge. Results show that minimum energy solutions achieve significant material as well as total energy (or carbon equivalent) savings compared with optimized passive structures.

This Research Topic has opened up a series of interesting new avenues. Consideration of material, energy and carbon costs is not only important for new construction but also to existing buildings and infrastructure. Most structures typically have a significant reserve capacity that is often not utilized before the end of service. Among possible future work, retrofitting active systems could be an effective means of ensuring safety and improving the behavior of aging structures. Structural adaptation could be employed to help diagnosis as well as to increase reserve capacity through active stress homogenization. Some of the methods described in this Research Topic could be repurposed for optimal retrofitting of sensing and actuation technologies to extend the service life of existing structures and in so doing deferring the disposal of embodied energy and carbon.

The work contained in this Research Topic demonstrates that current knowledge of methods for optimal integration of sensing and actuation technology in structures could lead to widespread adoption and large-scale applications. Adaptation enables new design and improved performance using less material, carbon and energy resources. For this reason, adaptive structures have great potential to reduce adverse environmental impacts caused by the construction industry.

\section{AUTHOR CONTRIBUTIONS}

GS curated this Research Topic with contribution of IS. GS was actively involved in reviewing all articles. GS wrote the first draft of the editorial. GS and IS contributed to the review, revised and approved the submitted version of the editorial.

\section{ACKNOWLEDGMENTS}

The editors would like to thank all contributors to this Research Topic as well as the reviewers and Frontiers Editorial Team.

Conflict of Interest: The authors declare that the research was conducted in the absence of any commercial or financial relationships that could be construed as a potential conflict of interest.

Copyright (C) 2021 Senatore and Smith. This is an open-access article distributed under the terms of the Creative Commons Attribution License (CC BY). The use, distribution or reproduction in other forums is permitted, provided the original author(s) and the copyright owner(s) are credited and that the original publication in this journal is cited, in accordance with accepted academic practice. No use, distribution or reproduction is permitted which does not comply with these terms. 